Annuaire suisse de politique de développement

11 | 1992

Annuaire Suisse - Tiers Monde 1992

\title{
Agrochimie, Suisse et Tiers Monde
}

\section{Vittorio Delucchi}

\section{OpenEdition}

\section{Journals}

Édition électronique

URL : http://journals.openedition.org/aspd/1567

DOI : $10.4000 /$ aspd. 1567

ISSN : 1663-9669

\section{Éditeur}

Institut de hautes études internationales et du développement

\section{Édition imprimée}

Date de publication : 1 janvier 1992

Pagination : 213-233

ISSN : 1660-5934

\section{Référence électronique}

Vittorio Delucchi, «Agrochimie, Suisse et Tiers Monde », Annuaire suisse de politique de développement [En ligne], 11 | 1992, mis en ligne le 18 mai 2013, consulté le 08 septembre 2020. URL : http:// journals.openedition.org/aspd/1567 ; DOI : https://doi.org/10.4000/aspd.1567 


\title{
Agrochimie, Suisse et Tiers Monde
}

\author{
Vittorio Delucchi
}

\section{Situation générale}

Les deux catégories de produits chimiques les plus utilisées en agriculture sont les engrais et les pesticides [définition: voir HAYES, 1991]. La Suisse en a exporté en 1990 pour une valeur d'env. 796 millions de francs et importé pour une valeur d'env. 230 millions [ANONYME, 1991]. Le commerce extérieur de ces produits agrochimiques s'est surtout développé avec les pays de la Communauté Européenne (CE) et de l'Association Européenne de libre Echange (AELE), toutefois de façon différente suivant les catégories. Ainsi, la Suisse a exporté 7 fois plus de pesticides qu'elle en a importé (env.790 millions de francs contre 113, (Tableau 1), mais elle a importé 21 fois plus d'engrais qu'elle en a exporté (env. 117 millions de francs contre 5,4).

Les importations d'engrais proviennent à raison de $54 \%$ de la France et de la Rép. Fédérale d'Allemagne (RFA) et à raison de $77 \%$ de ces deux pays plus le Bénélux. La participation des pays du Tiers Monde (Pérou, Chili, Trinité, Egypte, Tunisie, Nigéria, Arabie Séoudite, Malaisie) aux livraisons d'engrais à la Suisse n'excède point $2,3 \%$. Compte tenu de cette situation, la catégorie des engrais chimiques ne sera plus considérée par la suite. Cependant, il ne faut pas oublier que ces engrais ont, à long terme, un impact non négligeable sur la dynamique des populations d'organismes phytophages et sur l'environnement; leur action est toutefois plus lente et moins brutale que celle des pesticides. En vue d'augmenter la production agricole, les pays du Tiers Monde utiliseront des quantités d'engrais de plus en plus grandes et ces engrais seront fabriqués sur place. Par exemple, la production d'engrais en Inde devrait passer, d'après Krishna Murti [1989], de 5,25 millions de tonnes en 1984/85 à 19 millions de tonnes en ran 2000 et cette augmentation va poser d'importants problèmes écotoxicologiques. 
Tableau $N^{2} 1$

Commerce extérieur suisse des pesticides dans le monde pour l'année 1990, valeurs en francs suisses

\begin{tabular}{|l|c|r|}
\hline & & \\
Pesticides & Importations & Exportations \\
Insecticides & $25.148 .392 .-$ & $187.435 .784 .-$ \\
Fongicides & $29.952 .110 .-$ & $322.776 .201 .-$ \\
Herbicides & $36.201 .773 .-$ & $246.143 .700 .-$ \\
Désinfectants & $10.012 .482 .-$ & $18.201 .265 .-$ \\
Autres biocides & $11.811 .708 .-$ & $15.758 .357 .-$ \\
\hline Totaux & $\mathbf{1 1 3 . 1 2 6 . 4 6 5 . -}$ & $\mathbf{7 9 0 . 3 1 5 . 2 8 7 . -}$ \\
\hline
\end{tabular}

source : ANONYME, 1991

Le commerce extérieur suisse des pesticides touche, par contre, plus de cent pays sur tous les continents (cf. tableaux $n^{2} 2$ à $n^{0} 6$ : Annexe, pp. 227-231). II concerne surtout les insecticides, les fongicides et les herbicides (resp. $24 \%, 41 \%$ et $31 \%$ à l'exportation et $22 \%, 26,5 \%$ et $32 \%$ à l'importation). Les exportations ont lieu à raison de $55 \%$ vers les pays de la $\mathrm{CE}$ et de $65 \%$ vers les pays européens, y compris I'URSS. Les pays du Tiers Monde absorbent presque $30 \%$ des exportations suisses de pesticides. Le reste (env. $5 \%$ ) est exporté vers l'Amérique du Nord, l'Océanie et le Japon. Les importations de pesticides proviennent surtout des pays de la CE et de l'AELE, ainsi que des USA (total: 97\%); le Kenya, Israel, le Brésil, Taïwan, l'Inde et Hongkong sont pratiquement les seuls pays du Tiers Monde qui livrent des pesticides ou leurs composants, toutefois en quantité négligeable (moins de $3 \%$ ). Pour comprendre la place que la Suisse occupe dans le marché des pesticides, il est nécessaire d'en faire une analyse globale. (cf. tableau $n^{0} 7$, p. 231).

\section{Le marché des pesticides}

Sur le plan régional, le marché des pesticides est soumis aux hasards du temps atmosphérique (par ex., l'humidité élevée qui favorise le développement des maladies fongiques, ou l'hiver doux qui contribue à la survie des pucerons, ou encore des pluies abondantes qui réduisent l'utilisation des herbicides), du marché des produits agricoles (étendue des surfaces cultivés, par ex. de soya), de la politique agricole (subventions des pesticides et/ou des cultures), de la disponibilité en devises fortes et de l'inflation (pays du Tiers Monde) et d'autres facteurs aléatoires. Toutefois, sur le plan mondial, on remarque au fil des années une certaine stabi- 
lité marquée par une constante augmentation de la valeur de ce marché qui a été, en moyenne, de 4,1\% par an pendant la période 1972-90 [ANONYME, 1991b].

Le marché mondial des pesticides a atteint en 1990 une valeur estimée à US\$ 26,4 milliards, c'est-à-dire 33 à 38 milliards de francs suisses suivant le taux de change au cours de l'année. Les herbicides y occupent la meilleure place (44\%), suivis des insecticides $(29 \%)$, des fongicides $(21 \%)$ et des autres pesticides (rodenticides, nématicides, produits de fumigation, régulateurs de croissance des plantes, etc. : 6\%). La majorité des pesticides est appliquée à un nombre relativement petit de cultures: ainsi, on estime que l'arboriculture, la viticulture et l'horticulture absorbent $24 \%$ de la valeur marchande des pesticides, les céréales en absorbent $17,8 \%$, les rizières $11,7 \%$, les cotonneraies $10,3 \%$, les cultures de maïs $9,3 \%$, celles de soya $7,1 \%$, celles de betteraves à sucre $3,3 \%$, et le reste $16,5 \%$. Les pays industrialisés sont les principaux utilisateurs de pesticides: les pays de la CE et de l'AELE en consomment env. $28 \%$ de leur valeur marchande, les USA 25\% (en 1984: 34\%), le Japon env. 12\%, l'Europe de l'Est $9 \%$, et les pays du Tiers Monde env. 26\% [ANONYME, 1991b].

Si l'on ne tient pas compte de leurs effets indésirables, les pesticides ont été en agriculture le partenaire idéal des facteurs qui ont le plus contribué à l'augmentation rapide de la production agricole, tels la mécanisation (qui a favorisé la monoculture), les engrais chimiques, et la sélection de variétés de plantes à haut rendement. Les différentes catégories de pesticides n'ont toutefois pas connu le même développement, et la meilleure place occupée aujourd'hui par les herbicides est surtout due à l'augmentation du coût du travail et à la réglementation relativement peu sévère quant à leur utilisation. Un tiers des herbicides $(33,5 \%$, soit pour une valeur de US\$3,894 milliards) a été utilisé en 1990 aux USA, 26,3\% dans les pays de la CE et de l'AELE, et $9 \%$ au Japon. Les augmentations des ventes les plus élevées ont été enregistrées pour les diazines, les diphényl éthers, le glyphosate, et les nouveaux produits des groupes des imidazolinones et des sulphonyl urées, quoique les triazines, les amides et le carbamates (parmi les plus connus: Atrazine, Alachlor et Metolachlor, Thiotencarbe) restent à la tête du marché mondial; la valeur marchande de ces trois catégories d'herbicides est d'env. US\$ 4 milliards (ce qui fait à peu près un tiers de la valeur totale du marché des herbicides). Dans les seuls USA on applique $72,4 \%$ des herbicides utilisés mondialement en culture de soya, $61,7 \%$ de ceux utilisés en culture de maïs, et $37,4 \%$ des herbicides appliqués en cotonneraie, pour une valeur totale de US $\$$ 2,4 milliards. Le Japon utilise $60 \%$ des herbicides appliqués aux rizières, quoique la surface rizicole japonaise ne représente qu'1,4\% de la surface rizicole mondiale. Les pays de la CE et de l'AELE sont en tête en ce qui concerne la culture des betteraves à sucre $(62,7 \%$ des herbicides appliqués à cette culture), les céréales $(36 \%$, idem), l'arboriculture, la viticulture et l'horticulture $(33,6 \%$, idem). Comme le marché des herbicides se situe en grande partie dans les pays industrialisés, la recherche s'oriente plus particulièrement en fonction des problèmes qui existent aux USA, en Europe et au Japon [ANONYME, 1991b].

Contrairement à ce que l'on constate pour les herbicides, plus de la moitié des insecticides (acaricides compris) ont été vendus en 1990 en dehors de l'Europe et 
des USA. Les pays du Tiers Monde en ont acheté (ou reçu) pour env. $43 \%$ de leur valeur marchande (ce qui équivaut à US\$3,29 milliards), le Japon env. $12 \%$. Si on considère la répartition des insecticides par culture, on remarque que $71 \%$ des produits appliqués en arboriculture, viticulture et horticulture sont vendus au Japon, aux USA et en Europe de l'Ouest, que 61,5\% des insecticides appliqués en culture de coton sont utilisés en Inde, USA, URSS et Chine, que $70,7 \%$ des insecticides répandus en culture de maïs sont vendus aux USA, en Europe et en URSS, et enfin que le Japon achète plus d'un tiers des insecticides appliqués dans les rizières. Les produits les plus connus sont les organophosphorés (par ex. Parathion, Malathion, Phosphamidon), dont le chiffre d'affaires est estimé à $37 \%$ du marché mondial des insecticides, ensuite les carbamates (par ex. Carbaryl, Carbofuran, Méthomyl) et les pyréthrinoides (par ex. Perméthrine, Cyperméthrine, Deltaméthrine, Fenvarelate). Ces derniers ont connu un développement formidable au cours des 15 dernières années, au point que la valeur de leur marché était, en 1990, env. $50 \%$ de celle des organophosphorés [ANONYME, 1991b].

A la suite de conditions atmosphériques qui ont favorisé en 1990 le développement de maladies fongiques, l'Europe de l'Ouest est devenue le plus grand marché des fongicides, ces produits ayant atteint une valeur marchande de US\$ 2,611 milliards, soit $47,1 \%$ de la valeur du marché mondial. Les fongicides s'appliquent surtout aux cultures de céréales et à la viticulture (78\% resp. 50,3\% de la valeur totale des fongicides appliqués aux deux cultures dans le monde). Une autre tranche assez grosse de fongicides a rejoint les rizières japonaises $(51,7 \%$ du chiffre d'affaires des fongicides utilisés mondialement en rizière). Les triazoles (systémiques) ont été de loin les fongicides les plus vendus, surtout en Europe de l'Ouest (85\%) (parmi les plus connus: Bayleton, Tilt et Topas), suivis des dithiocarbamates (non systémiques), dont les plus connus sont le Maneb et le Mancozèbe (env. 2/3 du marché des dithiocarbamates). Triazoles et dithiocarbamates totalisent environ $32 \%$ des ventes des fongicides [ANONYME, 1991b].

En dehors des trois catégories de pesticides susmentionnées, il existe un marché assez restreint concernant les nématicides, les produits de fumigation et les régulateurs de croissance des plantes (au total: $6 \%$ du marché mondial). Les nématicides sont surtout utilisés en culture sous verre, les régulateurs de croissance trouvent leur emploi en cotonneraie (ouverture des capsules), en culture de céréales (contre la verse), en arboriculture (mise à fruit), en horticulture (suppression de la croissance), et comme défoliateurs (par ex. en culture de coton). $70 \%$ du marché des régulateurs de croissance des plantes se situe en Europe de l'Ouest et en Amérique du Nord. Les produits les plus connus sont l'hydrazide maléique, le Chlorméquat et l'Ethéphon [ANONYME, 1991b].

Les plus grands exportateurs de pesticides sont, par ordre d'importance, la RFA, les USA, la France, la Grande Bretagne, la Suisse, les Pays-Bas, et le Japon. La valeur des exportations de ces pays pris ensemble a atteint US $\$ 4,631$ milliards en 1988 et US $\$ 4,826$ milliards en 1989. Les données pour 1990 ne sont pas disponibles, mais devraient être du même ordre que pour l'année précédente. 
Tandis que la valeur des exportations a augmenté de 47,8\% entre 1979 et 1989, le volume (tonnage) des exportations des 7 pays cités a diminué de $21,8 \%$. Cela est dû en partie à l'augmentation de l'efficacité des produits. La Suisse a exporté en 1989 pour US $\$ 530$ millions de pesticides, ce qui constitue $11 \%$ du total des exportations des 7 pays susmentionnés. II n'a pas été possible de connaître le nom des produits qui ont été exportés.

\section{La toxicité des pesticides}

Les insecticides sont certainement les pesticides les plus dangereux. Les plus utilisés sont classés par l'OMS (1990) la, lb et II, la seule exception étant le Malathion qui est classé III ("slightly hazardous"). Les organophosphorés (OP) les plus connus, tels le Méthylparathion, le Phosphamidon et le Phorate, sont classés la, c'est-à-dire parmi les "extremely hazardous". Parmi les $\mathbf{4 8}$ pesticides classés la par l'OMS, 30 sont des OP et 25 sont des insecticides dont la LD50 $(\mathrm{mg} / \mathrm{kg}$, oralement chez les rats) n'excède pas 50 . Chez les pyréthrinoïdes et les carbamates la situation est légèrement meilleure, et les produits les plus vendus sont classés Ib "highly hazardous" et II "moderately hazardous". Les 4 pyréthrinoïdes qui dominent le marché - Fenvarelate, Perméthrine, Cyperméthrine et Deltaméthrine - sont classés II. Le Carbaryl est également classé II, tandis que le Méthomil et le Carbofuran sont rangés en classe lb et l'aldicarbe en classe la. Les carbamates sont très utilisés dans les cultures tropicales et sont en partie produits en Inde, à Costa Rica et en Extrême-Orient. Les organochlorés $(\mathrm{OC})$ sont encore autorisés dans beaucoup de pays et on estime que leur valeur marchande atteint aujourd'hui env. US $\$ 500$ millions. Parmi les OC qui dominent le marché on peut citer le Lindane, le Chlordane, le DDT, le Toxaphène, tous classés II, et l'Endrine, classée lb.

La toxicité des herbicides est, comparée à celle des insecticides, relativement faible. II n'y a aucun herbicide classé la et les quelques produits classés Ib sont très peu connus. Les triazines - telles l'Atrazine, la Simazine, la Prométryne et la Cyanazine - ainsi que la sulphonyl urée Chlorsulfuron (seul produit classé), les imidazolinones les plus vendus - tels I'Imazethapyr, I'Imazaquine et I'Imazamethabenz - et le glyphosate Roundup sont rangés parmi les produits "unlikely to present acute hazard in normal use" (pour faciliter la rédaction du texte, cette classe sera dénommé IV). On estime que la valeur marchande du seul Roundup en 1990 a été d'env. US\$ 950 millions. Dans ces catégories d'herbicides - triazines, sulphonyl urées, imidazolinones - il y a rarement des produits classés III. Malgré sa faible toxicité, la vente de l'Atrazine a été totalement défendue en Italie en 1990 et défendue dans le secteur agricole en RFA [ANONYME, 1991b]. Les thiocarbamates les plus vendus sont classés III, sauf quelques exceptions; les amides et les hormones sont classés III également, sauf le 2,4-D qui est classé II. La vente du paraquat (Gramazone), qui est un dérivé du bipyridylium (ou ammonium quaternaire) et dont l'usage est défendu dans plusieurs pays, a atteint en 1990 un chiffre d'affaires d'env. US $\$ 490$ millions. Les cas d'intoxication 
par herbicide concernent surtout le paraquat, qui est un produit peu sélectif classé II; comme il est inactivé dans le sol, il n'y a aucun effet résiduel. Le produit est toutefois dangereux pour l'homme du fait qu'il peut conduire à sa mort par asphyxie.

Le degré de toxicité des fongicides est également plus faible que celui des insecticides couramment utilisés. Les fongicides classés la et lb sont très rares. Les produits les plus vendus sont le Propiconazole, le Triadiméfon et le Pencazole ( $85 \%$ de la valeur des ventes des triazoles), qui sont classés II respectivement III et IV, ensuite le Mancozèbe et le Manèbe (2/3 de la valeur des ventes des thiocarbamates), le Bénomyle, le Carbendazine, le Thiabendazole et le Thiophanate méthyle (les plus vendus des benzimidazoles) qui sont classés IV, et enfin les produits cuivrés, très utilisés dans les pays du Tiers Monde et classés II et III, ainsi que les soufres, classés IV.

Les régulateurs de croissance des plantes, dont le chiffre d'affaires a atteint en 1990 US $\$ 550$ millions [ANONYME, 1991b], ont une toxicité relativement faible et sont classés III (par ex. le Chlorméquat) et IV (par ex. l'Ethéphon).

\section{Les conséquences indésirables}

Tout produit agrochimique cause des effets indésirables, dont l'importance dépend, d'une part, de sa toxicité et, d'autre part, du milieu dans lequel il est utilisé. Les engrais chimiques provoquent, eux aussi, des effets indésirables, mais ces effets se manifestent lentement, après de nombreuses années, et sont difficiles à saisir; les pesticides, par contre, ont une action beaucoup plus rapide et brutale, de sorte que les effets indésirables peuvent se manifester assez vite. Ces effets concernent surtout la santé humaine, l'environnement, et les aspects socioéconomiques.

\section{Santé humaine}

Les effets sur la santé humaine se manifestent d'abord chez les travailleurs agricoles et leurs familles. Comme les pesticides les plus toxiques sont en général les plus anciens et les meilleurs marchés, on les applique de préférence dans le Tiers Monde, c'est-à-dire dans un milieu ou l'agriculteur est souvent illettré et n'a aucun accès aux informations les plus élémentaires concernant la manipulation des poisons. Les produits sont souvent vendus par des représentants de maisons incompétents, préoccupés davantage de vendre que d'aider l'agriculteur à résoudre ses problèmes. Ce n'est donc pas surprenant qu'il y ait des cas d'empoisonnement. On brasse les bouillies avec les bras, on laisse traîner les emballages vides dans un coin de la ferme, on applique les bouillies avec les moyens du bord (dans les Hauts Plateaux des Andes à l'aide d'un seau et d'un balai!). Comme les agriculteurs méconnaissent tout danger dans la manipulation des pesticides, les traitements sont souvent appliqués par les jeunes enfants âgés de 10 à 15 ans et à des heures de la journée où la dérive est très forte. En Bolivie, une étude du 
Ministère de prévoyance sociale et de santé publique [ANONYME, 1990c] précise que $10,7 \%$ à $11,5 \%$ des personnes travaillant directement avec les pesticides ou en contact avec les résidus présentent une diminution de l'activité de la cholinestérase dans le sang, ce qui est indice d'intoxication. Ce taux a été observé en particulier chez les populations des vallées mésothermiques et des plaines orientales du pays, où l'agriculture est très développée. L'étude en question ne mentionne aucune mortalité due aux pesticides, mais certaines personnes interviewées ont assuré que les cas d'intoxication létale dus à la négligence (suicide exclu) ne sont pas si rares. Lois et règlements existent, mais personne ne les applique: les institutions gouvernementales n'ont ni les moyens ni le personnel compétent pour le faire. Dans les pays du Tiers Monde chacun a le droit de s'empoisonner et d'empoisonner n'importe qui.

Les estimations au niveau mondial des intoxications et des décès dus aux pesticides sont évidemment discutables, mais dans beaucoup de cas elles ont été évaluées par des comités d'experts. Ainsi, l'OMS considère qu'un million de cas d'intoxication accidentelle et 20 mille décès par an seraient la règle. D'autres auteurs attribuent aux pesticides une importance beaucoup plus grande et estiment qu'au moins 2,9 millions de cas d'intoxication et 200 mille décès devraient être attribués aux pesticides [LEVINE, 1990]. La plupart des décès est due au suicide. La cause des intoxications accidentelles est pratiquement toujours l'utilisation non conforme aux recommandations de l'industrie. Le lecteur trouvera plus de détails utiles dans HILL [1988]. Enfin, les pesticides ont des effets à long terme couvrant un éventail d'infirmités, tels le cancer, la stérilité, le diabète, la nécrose des os, la leucémie, des dégâts aux poumons, etc. [LEVINE, 1991]; ces effets sont toutefois assez rares.

Un autre aspect des effets sur la santé humaine est lié aux résidus de pesticides dans les produits agricoles. Dans les régions tropicales et subtropicales certaines cultures sont traitées jusqu'à la récolte, après avoir reçu un nombre assez élevé d'applications [MAGALLONA, 1980; BOTTRELL, 1983]. Tel est le cas de la culture de tomates qui, suivant le pays, peut être traitée chimiquement de 25 à 50 fois. Chez les animaux domestiques la situation n'est pas meilleure: ainsi on traite avec des désinfectants (EBS 3: contre les salmonelloses et les coccidioses) les aliments des volailles et on vend les oeufs sans aucun délai d'attente!

\section{Environnement}

Les effets sur l'environnement concernent aussi bien la contamination de certains éléments du milieu (persistance des produits dans le sol, pesticides dans l'eau potable et des rivières, transport de produits à longue distance par les courants aériens, etc.) que la perturbation des écosystèmes cultivés et sauvages par rupture des chaînes alimentaires et destruction des ennemis naturels des phytophages. La destruction de la faune utile provoque non seulement la résurgence des nuisibles, mais également l'apparition de nouveaux ravageurs qui étaient auparavant sous contróle biologique naturel. Les cochenilles et les espè- 
ces mineuses de plantes sont presque toujours tenues en échec par un complexe de parasitoïdes: les programmes phytosanitaires mal conçus provoquent la multiplication massive des premières et obligent à court terme à intensifier les applications chimiques.

II existe encore un marché estimé à US $\$ 500$ millions concernant les OC (DDT, Lindane et produits similaires) qui ont été interdits dans les pays industrialisés à cause de leur impact sur l'environnement. Ces produits sont relativement peu toxiques pour l'homme et les animaux supérieurs, sont persistants et bon marchés, et sont par conséquent exportés dans les pays du Tiers Monde. Le problème ne concerne pas exclusivement les pays utilisateurs, mais toute la communauté internationale à cause des résidus dans les produits agricoles exportés (végétaux et animaux) et du transport dans l'atmosphère. La Suède a été un des premiers pays à interdire le DDT il y a une vingtaine d'années et cela par solidarité internationale, puisque la contamination du sol suédois indiquait une dérive atmosphérique du pesticide dont le tonnage était 10 fois supérieur aux importations du pays.

\section{Problèmes socioéconomiques}

La sélection de populations de nuisibles résistantes aux pesticides est probablement l'effet le plus redouté par le paysan, surtout lorsque la résistance induite est croisée. La phytiatrie moderne est généreuse d'exemples dans ce domaine [KAY \& COLLINS, 1987]. Actuellement, on connaît au moins 590 espèces animales et végétales [GEORGHIOU, 1986] devenues résistantes à un ou plusieurs pesticides. Ce nombre inclut 447 espèces d'insectes et acariens, une centaine de pathogènes de végétaux, 41 espèces de mauvaises herbes, ensuite des nématodes et des rongeurs. Les cas les plus spectaculaires ont été observés en cotonneraie et en arboriculture, où la chute des rendements a obligé les agriculteurs à l'abandon des cultures. On se souviendra certainement de la résistance induite chez la mouche blanche Bemisia tabaci GENN. (Aleurode) dans les cultures de coton de la plaine de la Gézira (Soudan) au début des années ' 80 , qui a eu comme conséquence la dépréciation du lint suite à sa contamination par le miellat sécrété par l'insecte. On se souviendra également des cicadelles du riz devenues résistantes aux pesticides en Extrême-Orient et dans le Sud-Est asiatique: en Indonésie, au milieu des années ' 80 , il n'était plus possible de lutter chimiquement contre la cicadelle Nilaparvata lugens STAL qui menaçait la récolte chez $70 \%$ des rizières de Java (FAO, 1988). Les exemples de ce genre ne manquent pas. L'extension de la culture de soya en Amérique latine et, plus récemment, en Afrique pourrait conduire à une situation analogue. Dans la région bolivienne de Santa Cruz on applique déjà jusqu'à 5 traitements chimiques contre les insectes et les mauvaises herbes; compte tenu du prix des pesticides et de la concurrence sur le marché du soya, le paysan n'a presque plus aucune marge de bénéfice. Le jour où la culture ne sera plus rentable, il faudra bien revoir toute la conception phytosanitaire!

L'existence de résidus de pesticides dans les produits agricoles n'est pas seu- 
lement un problème d'hygiène, mais aussi socioéconomique. On rappellera ici un cas d'Amérique Centrale, où la viande a été refoulé à l'exportation à cause des résidus qu'elle contenait, le bétail ayant pâturé dans les zones limitrophes aux cultures de coton traitées aux pesticides [ICAITI, 1977; BOTTRELL, 1983]. Un exemple analogue concemant la viande de volailles est cité par HILL [1988].

Un autre aspect socioéconomique concerne la manipulation des pesticides. Dans certain pays du Tiers Monde, l'importateur achète des bidons (100-200 litres) ou des sacs (50-100 kg) de pesticide qu'il revend ensuite en bouteilles ou en sachets. Dans ces cas on ne connaît jamais la concentration en matière active et l'agriculteur peut appliquer un traitement inefficace lorsque le produit à été trop dilué. La même considération s'applique aux produits qui ont été mal conservés pendant trop longtemps. Dans les zones de frontière, les pesticides font l'objet de contrebande, de sorte que sur le marché apparaissent des substances dont l'emploi a été défendu.

Un dernier aspect socioéconomique concerne les subventions aux pesticides dans les pays du Tiers Monde (facilitations d'achat fournies par la Banque Centrale, comme en Bolivie; réduction du prix du traitement appliqué par des entreprises qui utilisent les pesticides obtenus gratuitement par le gouvernement, comme à Madagascar, etc.). Dans une étude conduite dans 9 pays du Tiers Monde [REPETTO, 1985] il a été remarqué que les subventions aux pesticides allaient de $15 \%$ à $90 \%$ avec une moyenne de $44 \%$ du prix de vente au détail. De ce fait les agriculteurs sont encouragés à utiliser les pesticides n'importe comment et abandonnent des techniques phytosanitaires traditionnelles valables. Dans le cas des pesticides (ou des cultures) subventionnés il devient difficile de calculer le seuil de tolérance du fait que les coûts de production sont faussés. Très souvent ce sont les grandes fermes qui profitent des subsides, car elles sont importatrices directes de pesticides. Le pesticide est comme la drogue: plus on en utilise et plus on en utiliserait. La quantité de pesticides vendus aux pays du Tiers Monde augmente chaque année sans que quelqu'un se préoccupe d'en évaluer les effets socioéconomiques négatifs [REPETTO, 1985].

\section{L'impuissance de la législation}

Le problème des exportations de pesticides vers les pays du Tiers Monde est délicat du fait qu'elles sont toujours mises en relation avec un autre problème: celui de l'insuffisance de la production agricole et de la faim. On part du principe que le seul moyen de résoudre cette insuffisance est le transfert des technologies utilisées dans les pays industrialisés, même de celles qui se sont révélées néfastes à la santé publique et à l'environnement.

Sur la base de simples considérations économiques à court terme on introduit la monoculture mécanisée et la méthode de lutte chimique sans trop se soucier des conséquences. HILL [1988] prétend en outre que $70 \%$ des pesticides exportés dans les pays du Tiers Monde sert à la production de biens d'exportation et non à résoudre le problème de la faim. Pour favoriser le marché tous les moyens 
sont bons. Toujours selon HILL [1988], 30\% des pesticides exportés des USA ne sont pas enregistrés auprès de l'Agence pour la Protection de l'Environnement [EPA] comme produits utilisables ou ne le sont plus du fait que leur vente à été interdite. On arrive aussi à court-circuiter la législation en exportant séparément les composants chimiques d'un produit non enregistré ou défendu. Contrairement au principe de jadis, on considère aujourd'hui que la sécurité est suffisante dans certains pays du Tiers Monde (Inde, Amérique Centrale, etc.) pour y installer des usines de pesticides: lindustrie y dépense beaucoup moins pour le contrôle de la pollution [HILL, 1988].

Beaucoup d'organisations, gouvernementales et non gouvernementales, se préoccupent depuis une vingtaine d'années de régler convenablement le problème de l'importation et de l'exportation de produits chimiques dangereux, toutefois avec peu de succès. D'un côté on se heurte aux intérêts économiques, de l'autre à l'ignorance du personnel phytosanitaire dans le Tiers Monde et à la corruption. HILL [1988] a donné un aperçu général de la situation et EGGER et PERROULAZ [1991] ont indiqué les difficultés qui existent avec le "principe de l'information et du consentement préalables" introduit en 1989, 4 ans après le code de conduite international sur la distribution et l'utilisation de pesticides [ADAM, 1987]. La Banque Mondiale [1990] a énoncé un certain nombre de règles applicables lors du financement de pesticides aux pays en développement, mais ces règles s'appuient sur la stratégie de la protection intégrée (PI), dont l'interprétation est assez difficile, même pour un phytiatre de chez nous. Cette stratégie à été introduite pour éliminer l'abus des pesticides; elle fait appel avant tout aux méthodes préventives (conservation des ennemis naturels des nuisibles, utilisation de variétés de plantes résistantes et de pratiques agronomiques adéquates), ensuite à la diversification des méthodes thérapeutiques (tactiques curatives). Le traitement chimique est appliqué d'après un seuil de tolérance, qui correspond à la densité d'un nuisible causant une perte de récolte supérieure au coût du traitement. Faute de recherches préalables, le seuil de tolérance est fixé empiriquement et, habituellement, trop bas. Ce qui est un moyen pour éliminer ou limiter un abus peut devenir, dans d'autres situations, un moyen pour intensifier la lutte chimique. Tout dépend par où commence l'interprétation de la PI. Pour cette raison on s'est préoccupé récemment de mieux définir les étapes qu'il faut considérer successivement lors de la mise sur pied d'un programme de PI [DELUCCHI, 1990]. Ces étapes sont décrites ici dans le cadre d'un projet qui a débuté à Madagascar en 1983 et dont la réalisation est financée par la Direction de la Coopération au Développement et de l'Aide humanitaire (DDA). Ce projet est un des rares exemples de gestion phytosanitaire des cultures en Afrique [PLI, 1985-1989].

\section{La protection intégrée des rizières à Madagascar}

Onze millions de malgaches consomment 2 millions de tonnes de paddy par an. Jusqu'au début des années ' 70 , la production de riz était suffisante et une partie 
était même exportée. Dix ans plus tard elle était déficitaire à cause de la dégradation des conditions de production, de transport et de communication, et de l'augmentation de la population. En 1982 les importations de riz à Madagascar atteignaient le niveau record de 342 mille tonnes. Le gouvernement lançait alors plusieurs opérations visant l'autosuffisance alimentaire. L'une d'entre elles consistait à appliquer des traitements chimiques aériens dans la cuvette du lac Alaotra, la plus importante région rizicole du pays (environ 80 mille ha de rizières, pratiquement en monoculture), et avait comme objectif la lutte contre le borer blanc africain (Maliarpha separatella RAG.). Ce borer était considéré l'ennemi numéro un des rizières malgaches. Le gouvernement suisse accepta de financer l'opération, en posant toutefois une condition: qu'un projet de recherche soit lancé afin d'identifier les vrais problèmes phytosanitaires des rizières [ZAHNER, 1989].

La première tâche du projet fut l'identification et la pondération des différents éléments qui constituent l'agroécosystème rizière et d'en connaître les interactions. Cette recherche portant sur la flore et la faune associées à la culture montra que la rizière malgache est très riche en espèces: parmi les seuls arthropodes, plus de 950 espèces furent capturées, dont certaines étaient connues ailleurs comme des ravageurs, en particulier les cicadelles qui, aussi bien au Japon que dans tout le Sud-Est asiatique, ont été les insectes les plus redoutés à l'époque de l'apparition des OP sur le marché.

Parallèlement à cet inventaire faunistique et floristique on procédait à l'évaluation de la nuisibilité des phytophages susceptibles de causer une perte économique, c'est-à-dire du borer blanc, du pou du riz (Dicladispa gestroi CHAP., un coléoptère), les rongeurs et des mauvaises herbes. Le borer ne cause une perte économique que si le taux d'infestation des tiges dépasse $30 \%$ et l'attaque a lieu entre la $6 e$ et la $10 e$ semaine après repiquage dans les rizières repiquées "à temps". Compte tenu du coût d'un traitement chimique, l'application d'un insecticide ne peut avoir lieu que lorsque le taux d'infestation est encore plus élevé, un niveau qui n'a jamais été atteint au cours des 7 dernières années. Le borer constitue donc au lac Alaotra un "cas limite" qu'il faut surveiller. L'étude de la nuisibilité du pou montra que la plante de riz supporte l'infestation d'au moins une larve par feuille sans qu'il y ait perte de rendement; en 1988, ce seuil était atteint chez 1,8\% des $\mathbf{2 7 0 0}$ foyers repérés au lac Alaotra. Le pou du riz constitue également un "cas limite" qu'il faut surveiller, une augmentation du taux d'infestation n'étant jamais exclue. La perte de rendement avant récolte causée par les rongeurs a été évaluée à moins de $1 \%$. Les mauvaises herbes - graminées et cypéracées - causent en moyenne une réduction du rendement de $25 \%$ et cette perte est d'autant plus élevée que la culture est intensive. Les recherches au lac Alaotra ont donc montré que les arthropodes et les rongeurs ne représentent aucun danger et que les traitements appliqués entre 1983 et 1985 étaient plus ou moins inutiles: des essais ont par la suite démontré que le Phosphamidon utilisé tue moins que 10\% des populations du borer.

Le problème phytosanitaire principal est donc constitué par les mauvaises herbes: cependant, un seul sarclage en début de tallage suffit pour supprimer toute action néfaste des mauvaises herbes, ces dernières n'étant plus nuisibles 
après la montaison. Contre le pou du riz on traite chimiquement les pépinières, où débute l'infestation, mais ces traitements sont ponctuels et concernent une surface très restreinte (environ $3 \%$ ): l'agroécosystème rizière n'est donc pas perturbé. Le borer blanc reste à longue haleine le seul danger potentiel; pour cela on a mis sur pied un service d'avertissement, mais à l'avenir le problème pourra être résolu en augmentant l'action des ennemis naturels et des entomopathogènes, dont le complexe est plus riche sur le continent africain. On pourra en même temps introduire des variétés de riz moins susceptibles aux attaques du borer, mais cela exige une surveillance constante du fait qu'elles peuvent favoriser le développement d'autres arthropodes jusqu'ici indifférents. La surveillance est un prix qu'il faut payer en $\mathrm{PI}$, un prix de loin moins élevé que la dépendance d'un pesticide, dont une seule application avait coûté en 1983 plus de 3 millions de francs suisses.

La PI est avant tout de la prévention: exploitation des ressources naturelles (les ennemis des nuisibles, la résistance de la plante aux nuisibles) et la mise en place de pratiques culturales qui affaiblissent les populations des nuisibles ou qui interfèrent avec leur comportement. II faut renforcer ces tactiques préventives et n'utiliser les pesticides que dans des cas extrêmes, de la même façon qu'on appelle les pompiers seulement lorsque la maison brûle. Les pesticides ne sont pas les seuls moyens curatifs existants: on dispose aujourd'hui d'un certain nombre de techniques alternatives (biologiques, biotechniques, mécaniques, etc.) qui ne perturbent pas les rizières et qui peuvent être utilisées comme tactiques de transition, en attendant de définir la cause qui a transformé un "indifférent" en nuisible.

\section{Les alternatives à la lutte chimique d'intérét commercial}

II n'est pas toujours facile de découvrir la cause qui a transformé un organisme "indifférent" en nuisible et il n'est pas toujours possible d'éliminer cette cause, une fois connue, du fait des contraintes économiques du système de production. Dans ces cas il faut utiliser des tactiques curatives (ou thérapeutiques), parmi lesquelles les biologiques et les biotechniques devraient avoir priorité absolue. Les succès obtenus par la méthode de lutte biologique sont bien connus et documentés. Dans le cas des mauvaises herbes, dont la lutte chimique a coûté en 1990 US $\$ 11,625$ milliards, la recherche a montré récemment qu'il existe de nombreux organismes pathogènes spécifiques qui peuvent être utilisés pour lutter contre elles sans aucun danger pour les cultures. II s'agit de champignons qui ne sont introduits qu'une fois, comme dans le cas de Puccinia chondrillina BUBAK \& SYDOW contre la mauvaise herbe Chondrilla juncea en Australie, ou bien de mycoherbicides, qui sont répandus dans les cultures comme n'importe quel pesticide chaque fois qu'il est nécessaire d'intervenir contre une mauvaise herbe déterminée. Collego ${ }^{\circledR}$ est un mycoherbicide à base de Colletotrichum gloeosporioides PENZ., dont une forme spéciale est pathogène pour la mauvaise herbe Aeschynomene virginica en culture de soya et de riz [EVANS, 1991]. L'industrie s'intéresse évidemment aux mycoherbicides, pour lesquels il existe un 
marché énorme, surtout dans les tropiques, même si la spécificité d'un produit représente pour l'industrie un grand désavantage.

Chez les arthropodes, les méthodes biologique et biotechnique de lutte sont l'enfant pauvre de la protection phytosanitaire, malgré les connaissances accumulées au cours d'un demi siècle. La production mondiale actuelle de préparations à base de Bacillus thuringiensis BERL. (Bt), qui sont les plus connues parmi les bioinsecticides, est de 3 à 4 mille tonnes, correspondant à un chiffre d'affaires de US $\$ 50$ à 60 millions, c'est-à-dire à $1 \%$ du chiffre d'affaires total des insecticides [BASSAND, 1990]. II existe aujourd'hui des préparations commerciales à base de Bt utilisables contre une trentaine d'insectes (lépidoptères, moustiques, etc.); l'amélioration des souches existantes par des procédés de génie génétique ouvre des perspectives intéressantes en lutte microbiologique [BASSAND, 1990]. Par ailleurs, on dispose de préparations commerciales à base de virus pour lutter contre des lépidoptères et une tenthrède, à base de champignons (Verticillium, Beauveria, Metarhizium ) contre les mouches blanches et les pucerons en serre respectivement contre d'autres nuisibles des cultures, à base de nématodes pour lutter contre certains ravageurs vivant dans le sol et à base de protozoaires (Nosema). Parmi les alternatives commercialisables pour combattre les arthropodes il faut citer encore la lutte biologique à l'aide d'insectes ou d'acariens, tels les Trichogramma, les Chrysopa, les Aphytis, les Phytoseiulus, etc., utilisés en plein champ et en serre, et les méthodes biotechniques à base de médiateurs chimiques de communication appliquées surtout en arboriculture et en viticulture, mais aussi dans les grandes cultures. La lutte biologique à l'aide d'arthropodes a donné des résultats excellents aussi contre les mauvaises herbes.

Dans le domaine de la phytopathologie, le développement d'alternatives à la lutte chimique à été beaucoup plus lent que dans les autres domaines de la phytiatrie. Au cours des dix dernières années on a quand même mis sur le marché une demi-douzaine de préparations pour lutter contre certains phytopathogènes dans le sol, mais 2 seulement d'entre elles ont été autorisées aux USA par l'EPA: la formulation qui contient la bactérie Pseudomonas fluorescens et celle à base de Agrobacterium radiobacter [LEWIS \& PAPAVIZAS, 1991].

\section{Le génie écologique}

Le génie écologique (= ecological engineering) n'est pas seulement le titre d'une nouvelle revue d'écotechnologie, dont le premier volume paraîtra en 1992, mais aussi un programme de restructuration de notre agriculture sur des bases qui tiennent compte davantage des nécessités réelles et des aspects écologiques. Les nécessités réelles sont liées au comportement de l'agriculteur qui devrait apprendre à être plus critique en ce qui concerne l'emploi de produits agrochimiques: c'est un problème de formation et d'information, surtout dans les pays du Tiers Monde, où les institutions publiques nationales sont dépourvues de tout moyen. Les aspects écologiques ont trait à la diversification des cultures et de leur environnement, à l'exploitation raisonnable des ressources naturelles 
(eau, ennemis naturels des nuisibles, etc.), à la conservation de la fertilité du sol (surtout contre l'érosion), et à l'utilisation de technologies à faibles intrants. Cela ne signifie point que l'agriculture doit rester ou devenir extensive, bien au contraire. En Europe Centrale on peut pratiquer une arboriculture plus ou moins sans insecticides, sans qu'il y ait diminution des rendements (qualité et quantité). Dans les rizières d'Indonésie gérées suivant les principes de la PI, les traitements insecticides ont été réduits de $90 \%$ et les rendements à l'ha ont augmenté de 6,1 à $7,4 \mathrm{t}$ [FAO, 1988]. En matière de gestion phytosanitaire, nos connaissances sont encore très limitées: c'est bien en grande partie à cause de notre ignorance que le marché des pesticides est de plus en plus florissant.

\section{Conclusion}

Dans le monde on produit pour environ US $\$ 26,4$ milliards de pesticides (estimation 1990). Environ 1/4 de cette production est utilisé dans les pays du Tiers Monde. La Suisse est le 5 e pays exportateur de pesticides (classement suivant la valeur monétaire) après la RFA, les USA, la France et la Grande Bretagne. Sa participation aux exportations a été de $11 \%$ en 1989 , pour une valeur de US $\$ 530$ millions, dont environ $30 \%$ vers les pays en voie de développement. Les produits exportés sont inconnus; cependant, ils ne devraient pas être différents de ceux qui sont exportés par les autres pays et comprendre aussi bien des pesticides très toxiques (comme le Phosphamidon) que des pesticides présentant peu de risques pour l'agriculteur et l'environnement. L'exportation de pesticides très toxiques vers les pays du Tiers Monde, où le paysan est souvent illettré et méconnaît les risques associés à la manipulation des poisons, constitue un problème qui est loin d'être résolu sur le plan international. La législation ne sert à rien si dans les pays du Tiers Monde le personnel compétent est absent ou n'a ni la formation adéquate, ni l'information qui lui permet de prendre des décisions valables. La stratégie de la protection intégrée suppose l'existence de données qui, en général, n'existent pas. Pour cette raison il faudrait intensifier la recherche phytiatrique en zone tropicale et subtropicale et créer en même temps les bases pour une agriculture durable. 
Tableau $\mathrm{N}^{\circ} 2$

ANNEXE

Commerce extérieur suisse des pesticides en Europe [incl. I'URSS] pour l'année 1990, valeurs en francs suisses

\begin{tabular}{|c|c|c|c|}
\hline Pays & Importations & Exportations & Subtotaux \\
\hline $\begin{array}{l}\text { RFA } \\
\text { France } \\
\text { Danemark } \\
\text { Grande Bretagne } \\
\text { Italie } \\
\text { Pays Bas } \\
\text { Espagne } \\
\text { Grèce } \\
\text { Portugal } \\
\text { Belgique/Luxembourg } \\
\text { Irlande } \\
\text { Autriche } \\
\text { Suède } \\
\text { Norvège } \\
\text { Finlande } \\
\text { RDA* } \\
\text { Tchécoslovaquie } \\
\text { Pologne } \\
\text { Hongrie } \\
\text { Bulgarie } \\
\text { Roumanie } \\
\text { Yougoslavie } \\
\text { URSS } \\
\text { Chypre }\end{array}$ & $\begin{array}{r}32.267 .654 .- \\
31.033 .340 .- \\
63.812 .- \\
9.369 .666 .- \\
5.209 .702 .- \\
8.450 .425 .- \\
597.916 .- \\
-.- \\
900 .- \\
4.147 .925 .- \\
390.758 .- \\
2.405 .633 .- \\
944.133 .- \\
-.- \\
99.030 .- \\
-.- \\
-.- \\
1.368 .- \\
18.687 .- \\
-.- \\
-.- \\
26.500 .- \\
-.- \\
-.-\end{array}$ & $\begin{array}{r}116.838 .831 .- \\
105.329 .600 .- \\
51.654 .977 .- \\
45.375 .446 .- \\
40.314 .308 .- \\
16.569 .208 .- \\
13.819 .996 .- \\
8.624 .432 .- \\
7.115 .350 .- \\
5.796 .179 .- \\
2.881 .782 .- \\
15.272 .915 .- \\
6.022 .238 .- \\
2.510 .467 .- \\
6.022 .238 .- \\
1.140 .053 .- \\
7.835 .995 .- \\
4.226 .541 .- \\
5.612 .479 .- \\
5.100 .418 .- \\
4.019 .760 .- \\
3.773 .149 .- \\
40.822 .694 .- \\
691.062 .-\end{array}$ & 414.320.109.- \\
\hline Totaux & 95.028.817.- & 512.944.231.- & \\
\hline $\begin{array}{l}\text { *jusqu'au } 2 \text { octobre } 19 \\
\text { source : ANONYME, }\end{array}$ & & & \\
\hline
\end{tabular}


Tableau $\mathrm{N}^{\circ} 3$

Commerce extérieur suisse des pesticides en Afrique pour l'année 1990, valeurs en francs suisses.

\begin{tabular}{|c|c|c|}
\hline Pays & Importations & Exportations \\
\hline Egypte &.-- & 17.523.087.- \\
\hline Soudan & $\because-$ & 11.301.156.- \\
\hline Libye & $\because-$ & 3.155.252.- \\
\hline Maroc &.- & 2.241.883.- \\
\hline Côte d'Ivoire &.- & 5.142.869.- \\
\hline Nigéria &.- & 4.475.182.- \\
\hline Ghana & $\because-$ & 1.760.486.- \\
\hline Cameroun &.-- & 3.239.642.- \\
\hline Afrique du Sud & 1.162.- & 3.378.440.- \\
\hline Zimbabwe & $\because-$ & 2.485.051.- \\
\hline Mozambique & $-{ }^{-}$ & 3.582.508.- \\
\hline Madagascar & $\because-$ & 1.458.154.- \\
\hline Tanzanie &.-- & 1.866.736.- \\
\hline Ethiopie & $\because-$ & 11.357.853.- \\
\hline Kenya & 183.478.- & 29.261.225.- \\
\hline Autres pays* &.-- & 3.923.531.- \\
\hline Totaux & 184.640.- & 69.817.955.- \\
\hline \multicolumn{3}{|c|}{$\begin{array}{l}\text { *Tunisie, Algérie, Sénégal, Mali, Gabon, Congo, Tchad, Zaïre, } \\
\text { Angola, Zambie, Malawi, La Réunion, Maurice, Rwanda, Bunundi, } \\
\text { Somalie, Ouganda. }\end{array}$} \\
\hline
\end{tabular}


Tableau $\mathrm{N}^{\circ} 4$

Commerce extérleur suisse des pesticides en Amérique pour l'année 1990, valeurs en francs suisses

\begin{tabular}{|c|c|c|c|}
\hline Pays & Importations & Exportations & Subtotaux \\
\hline $\begin{array}{l}\text { Canada } \\
\text { USA }\end{array}$ & $\begin{array}{r}\text { 425.- } \\
\text { 15.705.575.- }\end{array}$ & $\begin{array}{r}\text { 4.401.827.- } \\
\text { 4722939.- }\end{array}$ & 9.124.766.- \\
\hline Mexique & 253.- & 6.183.092.- & \\
\hline Guatemala & $\because-$ & 2.419.999.- & \\
\hline Nicaragua &.-- & 1.630.755.- & \\
\hline Costa Rica & $\because-$ & 1.911.607.- & \\
\hline Panama & $\because-$ & 2.431.034.- & \\
\hline Cuba & $\because-$ & 17.586.901.- & \\
\hline Guadeloupe & $\because-$ & 1.160.759.- & \\
\hline Rép. Dominicaine &.-- & 2.135.212.- & \\
\hline $\begin{array}{l}\text { Autres pays } \\
\text { d'Amérique centrale* }\end{array}$ & $\because-$ & 3.708.520.- & 39.197.879.- \\
\hline Paraguay &.-- & 3.486.298.- & \\
\hline Uruguay &.-- & 1.908.963.- & \\
\hline Argentine & $-\because$ & 6.284.308.- & \\
\hline Chili & $\because-$ & 4.055.925.- & \\
\hline Bolivie & $\because-$ & 1.538.930.- & \\
\hline Pérou &.-- & 2.527.674.- & \\
\hline Equateur & $\because-$ & 4.072.420.- & \\
\hline $\begin{array}{l}\text { Autres pays } \\
\text { d'Amérique du Sud }\end{array}$ & 962149.- & 1.397.516.- & 21.199.614.- \\
\hline Totaux & 16.667.902.- & 69.522.259.- & \\
\hline \multicolumn{4}{|c|}{$\begin{array}{l}\text { "Honduras, El Salvador, Jamaïque, Sainte Lucie, Barbade, } \\
\text { Martinique, Monserrat, Trinité et Tobago. } \\
\text { "*Colombie, Vénézuela, Guyane, Surinam, Brésil. }\end{array}$} \\
\hline \multicolumn{4}{|c|}{ Source : ANONYME, 1991} \\
\hline
\end{tabular}


Tableau $\mathrm{N}^{\circ} 5$

Commerce extérieur suisse des pesticides en Asie pour l'année 1990, [valeurs en francs suisses

\begin{tabular}{|l|r|r|}
\hline Pays & Importations & Exportations \\
\hline Turquie & -- & $13.126 .376 .-$ \\
Syrie & --- & $1.565 .379 .-$ \\
Liban & --- & $1.647 .147 .-$ \\
Israel & $761.332 .-$ & $3.123 .812 .-$ \\
Arabie Séoudite & --- & $8.083 .181 .-$ \\
Emirats arabes & --- & $1.211 .743 .-$ \\
Irak & -- & $2.164 .216 .-$ \\
Iran & --- & $28.891 .810 .-$ \\
Pakistan & --- & $9.124 .936 .-$ \\
Bangladesh & --- & $1.095 .341 .-$ \\
Thaïlande & --- & $7.686 .934 .-$ \\
Malaisie & --- & $1.840 .368 .-$ \\
Singapour & --- & $1.000 .466 .-$ \\
Chine & $176.618 .-$ & $6.736 .024 .-$ \\
Hongkong & $48.616 .-$ & $1.531 .220 .-$ \\
Taïwan & $49.980 .-$ & $3941.004 .-$ \\
Corée du Sud & -- & $1.392 .712 .-$ \\
Japon & $176.279 .-$ & $11.901 .082 .-$ \\
Philippines & $-\because-$ & $7.586 .054 .-$ \\
Autres pays* & $29.590 .-$ & $3.333 .478 .-$ \\
\hline Totaux & $1.065 .797 .-$ & $105.082 .201 .-$ \\
\hline
\end{tabular}

*Jordanie, Yemen [Nord], Qatar, Oman, Koweït, Inde, Sri Lanka, Viêt-Nam, Indonésie.

Source : ANONYME, 1991 
Tableau $\mathrm{N}^{\circ} 6$

Commerce extérieur suisse des pesticides en Océanie pour l'année 1990, valeurs en francs sulsses

\begin{tabular}{|c|c|c|c|}
\hline Pays & Importations & Exportations & Subtotaux \\
\hline $\begin{array}{l}\text { Australie } \\
\text { Nouvelle Zélande } \\
\text { Papouasie- } \\
\text { Nouvelle Guinée } \\
\text { Polynésie française } \\
\text { Total }\end{array}$ & $\begin{array}{l}\because- \\
\because- \\
\because- \\
-\because\end{array}$ & $\begin{array}{r}\text { 14.834.710.- } \\
1.698 .694 .- \\
\\
81.615 .- \\
65.473 .- \\
\\
16.680 .492 .-\end{array}$ & $\begin{array}{r}16.533 .404 .- \\
147.088 .-\end{array}$ \\
\hline
\end{tabular}

Tableau $\mathrm{N}^{\circ} 7$

Importance relative du commerce extérieur suisse des pesticides dans le monde sulvant les continents et le degré de développement industriel pour l'année 1990

(somme des + : total; somme des 0 : pays du Tiers-Monde)

\begin{tabular}{|c|c|c|c|c|c|}
\hline Régions & Importations en & & Exportatior & is en $\%$ & \\
\hline $\mathrm{CE}+\mathrm{AELE}$ & 81.00 & & 56.59 & & \\
\hline Europe, incl. URSS & 84.10 & & 65.47 & + & \\
\hline Asie, incl. Turquie & 0.94 & & 15.05 & + & \\
\hline Asie, sans Japon & 0.78 & $\circ$ & 13.52 & & $\circ$ \\
\hline Afrique & 0.16 & $\circ$ & 8.98 & + & $\circ$ \\
\hline Amérique du Nord & 13.90 & & 1.17 & + & \\
\hline Amérique Latine & 0.90 & $\circ$ & 7.42 & + & 。 \\
\hline Océanie &.-- & & 1.91 & + & \\
\hline \multirow{2}{*}{$\begin{array}{l}\text { Océanie, sans Australie } \\
\text { et sans Nouvelle Zélande }\end{array}$} & $\because-$ & $\circ$ & 0.002 & & \\
\hline & $100 \%$ & & $100 \% 2$ & $29.94 \%$ & \\
\hline
\end{tabular}




\section{Documents consultés}

Adam, A.V., "Code de conduite international sur la distribution et l'utilisation de pesticides", 389-394, dans Delucchi, V. (éd.), Protection intégrée: quo vadis ?, Parasitis 86, 411 pages, 1987.

Anonyme (a), Statistique du commerce extérieur de la Suisse, Statistique annuelle 1990, Direction générale des douanes, Berne, vol.1 (928 pages), vol.2 (668 pages), vol. 3 (212 pages), 8 février 1991.

Anonyme (b), Agrochemical Service, County NatWest Securities Limited incorporating Wood Mackenzie \& Co. Ltd., London, 180 pages, juin 1991.

Anonyme (c), Investigación sobre intoxicación de trabajadores agricolas por plaguicidas, Ministerio de prevision social y salud publica, Bolivia, 160 pages, 1990.

Banque Mondiale, Guidelines for the procurement of pesticides (draft), 10 pages (+ Operational directive: agricultural pest management, 16 pages), 1990.

Bassand, D., " Bacillus thuringiensis: une alternative biologique à la lutte chimique contre les insectes nuisibles", Bull. Sandoz, n 93, 26e année, 25-29, 1990.

Bottrell, D.G., "Social problems in the pest management in the tropics", Insect Sci. Application, vol. 4, 179-184, 1983.

Bouguerra, M., "Les pesticides et le Tiers Monde", La Recherche, vol. 17, 545$551,1986$.

Delucchi, V., "Phytomedizinische Visionen", Landwirtschaft Schweiz, vol. 3, 469474, 1990.

Delucchi, V., Le Balpag en Bolivie - Mission d'évaluation de l'aide de la Suisse à la balance des paiements de Bolivie, Rapport rédigé à l'intention de l'Otfice Fédéral des Affaires Economiques Extérieures, 30 pages, 1991.

Egger, M. \& Perroulaz, G., "Revue", Annuaire Suisse-Tiers Monde, page 21, 1991.

Evans, H.C., "Biological control of tropical grassy weeds", manuscript, 16 pages, en cours de publication, 1991.

FAO, Integrated Pest Management in Rice in Indonesia, Jakarta, 13 pages, 1988.

Georghiou, G.R., "The magnitude of the resistance problem", 14-43, dans Anonyme, Pesticide resistance - Strategies and tactics for management, Natl. Academy Press, Washington, D.C., 471 pages, 1986.

Hayes, W.J., "Introduction", 1-37, dans Hayes, W.J. \& Laws, E.R. (eds.), Handbook of pesticide toxicology, Academic Press, Inc., vol. 1, 496 pages, 1991.

Hill, R., "Problems and policy for pesticide exports to less developed countries", Natural Resources J., vol.28, 699-72I, 1988.

Holl, K., Daily,, G. \& Ehrlich, P.R., "Integrated Pest Management in Latin America", Environm. Conservation, vol. 17, 341-350, 1990. 
ICAITI, Estudio de las consecuencias ambientales y economicas des uso de plaguicidas en la producción de algodon de Centroamerica, Informe final de la 1a fase, Guatemala, 320 pages, 1975.

Kay, I. R. \& Collins, P. J., "The problem of resistance to insecticides in tropical insect pests", Insect Sci. Application, vol. 8, 715-721, 1987.

Kohn, G.K. \& Raab, S.J., "Safety considerations relating to the manufacture of agrochemicals in the U.S. and the Third-World", 529-549, dans Hodgson, E. \& Kuhr, R.J. (eds.), Safer insecticides, M. Dekker, Inc., New York, 593 pages, 1990. Krishna Murti, C.R., "Chemicals in tropical and arid regions", 97-136, dans Bourdeau, Phj., Haines, J.A., Klein, W. \& Krishna Murti, C.R. (eds.), Ecotoxicology and climate, Scope 38, IPCS Joint Symposia 9, John Wiley \& Sons, 392 pages, 1989.

Levine, R., "Recognized and possible effects of pesticides in humans", 275-360, dans Hayes, W.J. \& Laws, E.R. (eds.), Handbook of pesticide toxicology, vol. 1, 496 pages, 1991.

Lewis, J.A. \& Papavizas, G.C., "Biocontrol of plant diseases: the approach for tomorrow", Crop Protection, vol. 10, 95-105, 1991.

Magallona, E.D., "Pesticide residues and relevant directions for developing countries", Residue Reviews, vol. 73, 13-26, 1980.

OMS, International Programme on Chemical Safety. The WHO recommended classification of pesticides by hazard and guidelines to classification 1990-1991, WHO/PCS/90.1 Rev 1, 39 pages, 1990.

PLI, Protection intégrée en riziculture au lac Alaotra, Rapports d'activité 1 à 5 , Zürich-Ambohitsilaozana, 1985 - 1989.

Reed, W., "Pest management issue - Food crops in developing countries", Brighton Crop Protection Conference, 289-295, 1988.

Repetto, R., Paying the price: pesticides subsidies in developing countries, World Resources Institute, Washington, D.C., 27 pages, 1985.

Schulten, G. G. M., "The role of FAO in IPM in Africa", Insect Sci. Application, vol. 10, 795-807, 1989.

Smith, E. H., "Integrated Pest Management (IPM) - Specific needs of developing countries", Insect Sci. Application, vol. 4, 173-177, 1983.

Zahner, Ph., "Protection intégrée en rizières à Madagascar - Possibilités et limites", 191-208, dans Bachmann, M. (ed.), Alimentation et développement, Zürich, 351 pages, 1989.

Zelazny, B., Chiarappa, L. \& Kenmore, P., "Integrated pest control in developing countries", FAO Plant Prot. Bull., vol. 33, 147-158, 1985. 